\title{
Diabetes Mellitus: could the Inhibition of a Single Enzyme (CPT-1) Involved in the Beta-Oxidation Process Improve this Complex Disease?
}

\author{
Piero Chiodi* \\ Freelance, Italy \\ Submission: December 2, 2016; Published: January 10, 2017 \\ *Corresponding author: Piero Chiodi, Freelance, Rome, Italy, Tel: +393475975307; Email: piero.chiodi@gmail.com
}

Keywords: CPT1; CPT inhibition; Ureidic compound; Discontinued development

Abbreviations: NIDDM: Non-Insulin-Dependent Diabetes Mellitus, FFA: Free-Fatty Acid; TG: Triglyceride

\section{Letter to Editor}

Diabetes mellitus is a heterogeneous chronic disease characterized by hyperglycemia, and insulin resistance. The causes lie the interaction of genetic and environmental factors that occur with deficiency in production of insulin by the pancreas, or by ineffectiveness of insulin produced.

Diabetes mellitus is among ten leading cause of death in high-income countries and it is increasing rapidly in countries undergoing industrialization. Diabetes alone claims on the average around $8 \%$ of total health budgets in developed countries [1]. Complications include diabetic retinopathy, renal failure, heart disease, diabetic neuropathy and foot ulceration and amputation. More than $90 \%$ of diabetic patients in the United States are Type 2 diabetics, or non-insulin-dependent diabetes mellitus (NIDDM). In NIDDM a severe glycemic control should be made by a synergic work of physical activity, diabetic diet, and the use of hypoglycemic agents, and lastly insulin.

Current hypoglycemic therapies are continuously ongoing, but not sufficiently adequate to control hyperglycemia, due to their side effect that discontinuous the treatment in the patients. It is possible distinguish different types of hypoglycemic agents: sulphonylureas (insulin secretagogues), biguanide, like metformin (insulin sensitizer and hepatic glucose production inhibitor), "glitazones" (insulin sensitizers), $\alpha$-glucosidase intestinal inhibitors (acarbose), meglitinides (glinides) (post prandial hypoglycemic agent) and ultimate metabolic hormones: GLP-1 receptor agonists (incretin mimetics) and DPP-4 inhibitors, both members of the glucagon peptide superfamily.
In the last decades, another pathway was tested in NIDDM to keep glycaemia and insulin resistance under control, I mean the way of limitation of mitochondrial fatty acid oxidation by CPT inhibition. It is known that fatty acids oxidation stimulates liver production of glucose, which is an important factor in diabetic hyperglycemia. The mechanism through which fatty acid oxidation stimulates hepatic glucose neo-synthesis is essentially by activation of pyruvate carboxylase in the mitochondrial matrix which requires acetyl-CoA, a major product of mitochondrial beta-oxidation of long-chain fatty acids, as activator to catalyzes the thermodynamically irreversible reaction, which is the rate limiting in hepatic gluconeogenesis [2].

In the past years, several approaches were attempted to inhibit the fatty acid oxidation pathway indirectly by inhibiting substrate release (fatty acids) from adipose tissue [3]. These approaches were unsuccessful because of side effects and/or lack of efficacy. Therefore, a different strategy was developed: the idea was to modulate liver fatty acid oxidation through the inhibition of the carnitine-dependent transfer of fatty acid from the cytosol to the mitochondrial matrix, where beta-oxidation occurs. Since beta-oxidation occurs in mitochondria, and fatty acids per se cannot pass the inner mitochondrial membrane, an important rate-limiting step in beta-oxidation exist: the pivotal enzyme CPT (CPT1, outer and CPT2, inner mitochondrial membrane). Its blockade can significantly reduce both the betaoxidation and glucose synthesis. Consequently, the inhibition of hepatic CPT1 can be considered an efficacious strategy in the therapy of diabetes, furthermore the condition of insulin 


\section{Current Research in Diabetes \& Obesity Journal}

resistance is evidenced by over-production of glucose in liver and under-utilization of glucose in muscle [4]. In addition, in NIDDM patients not adequately treated, increased gluconeogenesis was shown to be one of the major factors responsible for fasting and post-absorptive hyperglycemia [5].

Experimental observations of Jenkins and Griffith [6] showed the possibility of using DL-aminocarnitine a sliver CPT1 inhibitor. Infact, a strong hypoglycemic effect in fasted diabetic mice treated with a single dose $(0.3 \mathrm{mmol} / \mathrm{kg})$ was found plasma glucose levels normalized within 4-8 hours and remaining effective for at least $12 \mathrm{hr}$. However, in the previous experiment [7] when were administered a single dose ( $5 \mathrm{mmol} / \mathrm{kg}$ ) of acetylDL-aminocarnitine in starved mice it was found seriously liver and kidney triglyceride levels very markedly elevated for up to 3 days. Another potent inhibitor of CPT1 was then applied in experimental condition to correct glycaemia: etomoxir, ethyl (2[6(4-chloro-phenoxy)hexyl]oxirane-2-carboxylate) [8].

In a paper published in Diabetes, Reaven, Chang \& Hoffman [9] reported the effect of etomoxir on plasma glucose, freefatty acid (FFA), and triglyceride (TG) concentrations in rats with streptozocin-induced diabetes. The results were: lowering plasma glucose levels without affecting plasma insulin concentrations, with an increase in plasma FFA and TG concentrations, suggesting that modulation of FFA metabolism at the level of adipocytes or of the liver can have dramatic effects on carbohydrate and lipid metabolism [9].

A few years later, Hübinger, Weikert, Wolf \& Gries published a paper [10] in which etomoxir were considered as "a new therapeutic approach in diabetes" by inhibition of CPT1. To support this claim, they administered etomoxir in 8 type 2 diabetic patients drawing a placebo-controlled, double-blind randomized study by using the euglycemic clamp technique. Results were: the mean metabolic clearance rate of glucose was raised while free fatty acids plasma levels, glucose counter regulatory hormones, lipids and C-peptide values were unaffected. Due to these hopeful results, the scientific community expected more papers published on diabetic patients treated and/or the product launch as oral hypo-glycemic agent. The common element among the compounds above mentioned are irreversible inhibitors of CPT1 and they had not the selectivity of inhibition between two isoforms of CPT1, hepatic and cardiac, therefore, a greatest hitch in product development could be the accumulation of triglycerides in the heart (cardiac steatosis). To prevent this issue around the 2000s, it was proposed new compounds as potential antidiabetic agents able to perform that task [11].

In J Med Chem [12] we published a paper where we demonstrated in intact rat liver mitochondria the highest activity $(\mathrm{IC}(50)=0.7$ microM) for a compound belonging to the derived class of "carnitine analogues with long alkyl chain". In enzymatic inhibition tests, carried out in liver and heart mitochondria this compound also showed a good selectivity
$(\mathrm{M}-\mathrm{CPT} 1 / \mathrm{L}-\mathrm{CPT} 1 \mathrm{IC}(50)$ ratio $=4.86)$ for $\mathrm{CPT} 1$ between liver and heart isoform. This compound showed also a significant reduction of serum glucose levels in diabetic $\mathrm{db} / \mathrm{db}$ mice treated orally. Another compound we tested in experimental diabetes, an ureidic derivative from class of "carnitine analogues with long alkyl chain". This ureidic compound, with extraordinarily high selectivity toward the liver CPT1 isoform with respect to the heart isoform was able to reduce serum glucose levels in diabetic $\mathrm{db} / \mathrm{db}$ mice treated orally for 45 days at a dose of 50 $\mathrm{mg} / \mathrm{kg}$ twice a day, showing also an antiketotic activity in normal fasted rats and therefore selected for development as a potential antiketotic and antidiabetic drug [13].

Several years later were published by Conti, et al. [14] in Diabetes a paper where glucose production was investigated in isolated hepatocytes and during pancreatic clamps in healthy rats treated with the ureidic compound. In the same article was also investigated glucose metabolism and insulin sensitivity on $\mathrm{C} 57 \mathrm{BL} / 6 \mathrm{~J}, \mathrm{db} / \mathrm{db}$, high-fat fed mice, and rats after chronic treatments. The conclusions were that the ureidic compound, in vitro and in animal models, reduces gluconeogenesis and improves glucose homeostasis, refreshing the interest in selective and reversible L-CPT1 inhibition as a potential antihyperglycemic approach.

After exposure of these encouraging results and by-passed the obstacle of irreversibility and selective inhibition of CPT1 alone, the question is: why no pharmaceutical company has invested resources in this direction to make it a new antidiabetic drug? Perhaps, because of side effects this type of pharmacological approach on diabetes control could result blocking selectively liver CPT1 isoform; in fact, this isoform is also implicated in CNS control of food intake [15]. In addition, some negative aspects were already highlighted in previous studies such as steatosis [16] and apoptosis $[17,18]$; the latter aspect as to generate a new stream of research to anticancerogenic action due to CPT1 inhibition $[19,20]$. Moreover, other dangerous negative aspects of CPT blocking such as cardiac mitochondrial toxicity [21], cardiac hypertrophy $[22,23]$ and mortality for prolonged inhibition [24] emerged in other studies; consider that diabetes is a chronic disease and as such should be treated, the continued blockade of the CPT may be contraindicated? In contrast, some evidence had emerged from other CPT inhibitors to ameliorate or not effect on cardiac performance as result of inhibition $[11,24,25]$. In addition, a recent report showed that a long-term increase in hepatic fatty acid oxidation leads to a beneficial effect in a mouse model of obesity and diabetes [26]. The fact is that clinical studies on CPT inhibitor were discontinued. In conclusion, we can relaunch the Arduini's provocation by placing the problem: is inhibition or activation preferable? $[27,28]$.

\section{References}

1. Press Release on Global Burden of Diabetes (1998). World Health Organization Geneva.

2. Groen AK, van Roermund CW, Vervoorn RC, Tager JM (1986) Control of gluconeogenesis in rat liver cells. Flux control coefficients of the 


\section{Current Research in Diabetes \& Obesity Journal}

enzymes in the gluconeogenic pathway in the absence and presence of glucagon. Biochem J 237(2): 379-389.

3. Worm D, Henriksen JE, Vaag A, Thye-Ronn P, Melander A, et al, (1994) Pronounced blood glucose-lowering effect of the antilipolytic drug acipimox in noninsulin-dependent diabetes mellitus patients during a 3-day intensified treatment period. J ClinEndocrinolMetab 78(3): 717 721

4. DeFronzo RA, (1988) The triumvirate: beta-cell, muscle, liver. A collusion responsible for NIDDM. Diabetes 37(6): 667-687.

5. DeFronzo RA, Ferrannini E, Simonson DC (1989) Fasting hyperglycemia in non-insulin-dependent diabetes mellitus: contributions of excessive hepatic glucose production and impaired tissue glucose uptake. Metabolism 8(4): 387-395.

6. Jenkins DL, Griffith OW (1986) Antiketogenic and hypoglycemic effects of aminocarnitine and acylaminocarnitines. Proc Natl Acad Sci 83(2): 290-294.

7. Jenkins DL, Griffith OW (1985) DL-aminocarnitine and acetyl-DLaminocarnitine. Potent inhibitors of carnitine acyltransferases and hepatic triglyceride catabolism. J Biol Chem 260(27): 14748-14755.

8. Lopaschuk GD, Wall SR, Olley PM, Davies NJ (1988) Etomoxir, a carnitine palmitoyltransferase I inhibitor, protects hearts from fatty acid-induced ischemic injury independent of changes in long chain acylcarnitine. Circ Res Dec 63(6): 1036-1043.

9. Reaven GM, Chang H, Hoffman BB (1988) Additive hypoglycemic effects of drugs that modify free-fatty acid metabolism by different mechanisms in rats with streptozocin-induced diabetes. Diabetes $37(1): 28-32$.

10. Hübinger A, Weikert G, Wolf HP, Gries FA (1992) The effect of etomoxir on insulin sensitivity in type 2 diabetic patients. HormMetab Res 24(3): 115-118.

11. Deems RO, Anderson RC, Foley JE (1998) Hypoglycemic effects of a novel fatty acid oxidation inhibitor in rats and monkeys. Am J Physiol 274(2 Pt 2): R524-R528.

12. Giannessi F, Chiodi P, Marzi M, Minetti P, Pessotto P, et al. (2001) Reversible carnitine palmitoyltransferase inhibitors with broad chemical diversity as potential antidiabetic agents. J MedChem J 44(15): 2383-2386

13. Giannessi F, Pessotto P, Tassoni E, Chiodi P, Conti R, et al. (2003) Discovery of a long-chain carbamoyl aminocarnitine derivative, a reversible carnitine palmitoyltransferase inhibitor with antiketotic and antidiabetic activity. J MedChem 46 (2): 303-309.

14. Conti R, Mannucci E, Pessotto P, Tassoni E, Carminati P, et al. (2011) Selective reversible inhibition of liver carnitine palmitoyl-transferase 1 by teglicar reduces gluconeogenesis and improves glucose homeostasis. Diabetes 60(2): 644-651.

15. Gao S, Serra D, Keung W, Hegardt FG, Lopaschuk GD (2013) Important role of ventromedial hypothalamic carnitine palmitoyltransferase-1a in the control of food intake. Am J Physiol Endocrinol Metab 305(3): E336-E347.
16. Aires CC, Ijlst L, Stet F, Prip-Buus C, de Almeida IT, et al. (2010) Inhibition of hepatic carnitine palmitoyl-transferase 1 (CPT1A) by valproyl-CoA as a possible mechanism of valproate-induced steatosis. BiochemPharmacol 79 (5): 792-799.

17. de Pablo MA, Susin SA, Jacotot E, Larochette N, Costantini P, et al (1999) Palmitate induces apoptosis via a direct effect on mitochondria. Apoptosis 4(2): 81-87.

18. Henique C, Mansouri A, Fumey G, Lenoir V, Girard J, et al. (2010) Increased mitochondrial fatty acid oxidation is sufficient to protect skeletal muscle cells from palmitate-induced apoptosis. J Biol Chem 285(47): 36818-36827.

19. Ricciardi MR, Mirabilii S, Allegretti M, Licchetta R, Calarco A, et al. (2015) Targeting the leukemia cell metabolism by the CPT1a inhibition: functional preclinical effects in leukemias. Blood 126(16): 1925-1929.

20. Pucci S, Zonetti MJ, Fisco T, Polidoro C, Bocchinfuso G, et al. (2016) Carnitine palmitoyl transferase-1A (CPT1A): a new tumor specific target in human breast cancer. Oncotarget 7(15): 19982-19996.

21. Sayed-Ahmed MM, Shouman SA, Rezk BM, Khalifa MH, Osman AM, et al. (2000) Propionyl-L-carnitine as potential protective agent against adriamycin-induced impairment of fatty acid beta-oxidation in isolated heart mitochondria. Pharmacol Res 41(2): 143-150.

22. He L, Kim T, Long Q Liu J, Wang P, et al. (2012) Carnitine palmitoyltransferase-1b deficiency aggravates pressure overloadinduced cardiac hypertrophy caused by lipotoxicity. Circulation 126(14): 1705-1716.

23. Cabrero A, Merlos M, Laguna JC, Carrera MV (2003) Down-regulation of acyl-CoA oxidase gene expression and increased NF-kappaB activity in etomoxir-induced cardiac hypertrophy. J Lipid Res 44(2): 388-398.

24. Haynie KR, Vandanmagsar B, Wicks SE, Zhang J, Mynatt RL (2014) Inhibition of carnitine palymitoyltransferase1b induces cardiac hypertrophy and mortality in mice. Diabetes ObesMetab 16(8): 757 760.

25. Zhang Y, Fang X, Dai M, Cao Q, Tan T, et al. (2016) Cardiac-specific down-regulation of carnitine palmitoyltransferase-1b (CPT-1b) prevents cardiac remodeling in obese mice. Obesity (Silver Spring) (12): 2533-2543

26. Okere IC, Chandler MP, McElfresh TA, Rennison JH, Kung TA, et al (2007) Carnitine palmitoyl transferase-1 inhibition is not associated with cardiac hypertrophy in rats fed a high-fat diet. Clin Exp Pharmacol Physiol 34(1-2): 113-119.

27. Orellana-Gavaldà JM, Herrero L, Malandrino MI, Pañeda A, Sol Rodríguez-Peña $\mathrm{M}$, et al. (2011) Molecular therapy for obesity and diabetes based on a long-term increase in hepatic fatty-acid oxidation. Hepatology 53(3): 821-832.

28. Arduini A, Bonomini M (2011) Carnitine palmitoyltransferase as a potential target for treating diabetes: is inhibition or activation preferable? Hepatology 54(3): 1110-1111.

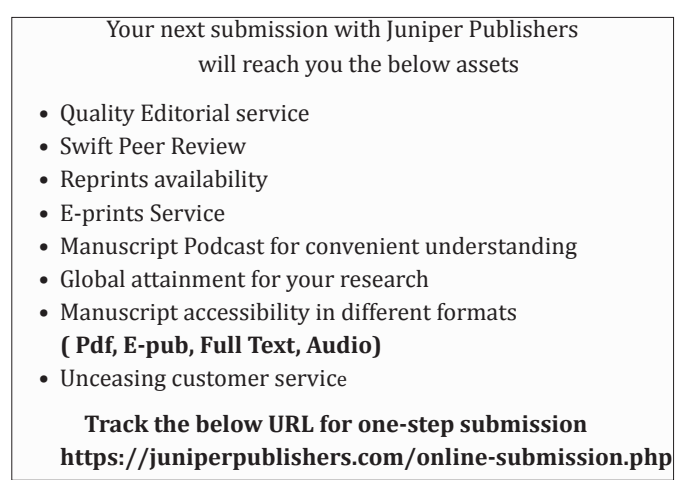

